\title{
Atti $5^{\circ}$ Congresso SISEF: Foreste e Società - Cambiamenti, Conflitti, Sinergie (a cura di: E. Lingua, R. Marzano, G. Minotta, R. Motta, A. Nosenzo, G. Bovio) \\ Qualità del fusto in un popolamento di rovere (Quercus petraea (Matt.) Liebl.) in Toscana
}

\section{Grifoni F}

v.le G. Matteotti, 19/D, I-52100 Arezzo (Italy) - email: fragrifon@tin.it.

\begin{abstract}
Stem-quality of sessile oak (Quercus petraea (Matt.) Liebl.) trees, Tuscany, Italy. The Regional Forest Inventory reports a significant presence of sessile oak in Tuscany. The noticeable increase of sessile oak timber value in better-shaped stems, suggests the advance evaluation of the quality of standing trees. In this way, the consistency of the applied silvicultural rules may be verified, too. The research trial was undertaken in a stand aged 41 to 45 originated from reafforestation a few hectares wide, located at Monte Lignano (Arezzo). The stand structure is not homogeneous as for tree size and tree species composition. Qualitative field surveys were carried out into three well-discernible stand types within the reforested area: a) scattered sessile oak trees into a withered chestnut orchard; b) pure sessile oak stands; c) scattered sessile oak trees into a poor chestnut coppice. Data collected at about one half of the foreseen stand life span showed the different stem quality into each structure and type. In addition, a diffuse growth of epicormic branches along sessile oak stems was observed into pure stands (b), following main crop thinnings and subordinate layer exploitation. This practice consisted therefore in a worsening of oak stems quality. As already showed by the practice of silviculture into sessile oak stands across Europe, these results proved that the production of valuable stems needs suited and well-timed tending practices.
\end{abstract}

Keywords: Sessile oak, Silviculture, Stem quality, Standing trees, Tuscany.

Received: Mar 31, 2006 - Accepted: Jan 01, 2007.

Citation: Grifoni F, 2007. Qualità del fusto in un popolamento di rovere (Quercus petraea (Matt.) Liebl.) in Toscana. Forest@ 4 (1): 126-130. [online] URL: http://www.sisef.it/.

\section{Introduzione}

Dall'elaborazione dei dati dell'Inventario Forestale della Toscana (Hofmann et al. 1998) si desume una discreta presenza della rovere nei diversi tipi di bosco della regione. La valenza di questa specie è accresciuta dalla qualità del legno prodotto ed è, quindi, opportuno analizzare i soprassuoli toscani con presenza di rovere, valutando le caratteristiche qualitative del fusto; queste possono essere influenzate da diversi fattori, fra i quali assumono particolare rilievo le scelte selvicolturali e la loro corretta esecuzione.

Obiettivo di questa indagine è proprio la valutazione qualitativa di piante di rovere presenti in differenti soprassuoli avviati ad alto fusto, nella fase intermedia di sviluppo, per acquisire conoscenze utili a definire una selvicoltura maggiormente mirata alla valorizzazione della produzione legnosa della specie, come già accade in Europa.

\section{Materiali e metodi}

L'indagine ha interessato un popolamento di alcuni ettari, localizzato sul Monte Lignano (Arezzo) fra 550 e 600 m s.l.m., originato da un rimboschimento effettuato nei primi decenni del 1900, poi sottoposto ad utilizzazioni a ceduo per la produzione di frasca da foraggio e legna da ardere. Le caratteristiche climatiche della zona, solo indicative per la distanza e la diversa altitudine dell'area sperimentale, sono riferite alla stazione termopluviometrica di Arezzo. I dati evidenziano un clima da umido a sub-umido, con moderata deficienza idrica in estate; la temperatura media annua è di $14^{\circ} \mathrm{C}$, la piovosità media an- 
Tab. 1 - Caratteri stazionali e dei soprassuoli analizzati.

\begin{tabular}{|c|c|c|c|c|c|c|c|c|}
\hline Tesi & Tipo soprassuolo & Particella & $\begin{array}{l}\text { Quota } \\
\text { (m s.l.m.) }\end{array}$ & $\begin{array}{l}\text { Esposi- } \\
\text { zione }\end{array}$ & $\begin{array}{l}\text { Pendenza } \\
\text { (gradi) }\end{array}$ & Età (anni) & $\begin{array}{c}\text { Piante di } \\
\text { rovere } \\
(\mathrm{n} / \mathrm{ha})\end{array}$ & $\begin{array}{l}\text { Altezza } \\
\text { dominante } \\
\text { (m) }\end{array}$ \\
\hline \multirow[t]{2}{*}{$\mathrm{A}$} & perticaia di rovere $\mathrm{e}$ & 1 & 550 & $\mathrm{~N}-\mathrm{NW}$ & 27 & 45 & 278 & 19.6 \\
\hline & $\begin{array}{l}\text { piante di castagno } \\
\text { da frutto }\end{array}$ & 2 & 550 & W - NW & $8-16$ & 41 & 375 & 15.8 \\
\hline \multirow[t]{3}{*}{ B } & perticaia rovere & 3 & 550 & $\mathrm{~N}$ & $20-25$ & 41 & 1125 & 14.6 \\
\hline & pura & 4 & 560 & $\mathrm{~N}-\mathrm{NW}$ & 17 & 41 & 1225 & 13.2 \\
\hline & & 5 & 570 & $\mathrm{~N}-\mathrm{NE}$ & $12-14$ & 41 & 1289 & 13.2 \\
\hline $\mathrm{C}$ & $\begin{array}{l}\text { perticaia di rovere e } \\
\text { ceduo di castagno }\end{array}$ & 6 & 600 & $\mathrm{~N}-\mathrm{NW}$ & 32 & 45 & 347 & 13.4 \\
\hline
\end{tabular}

nua è di $865 \mathrm{~mm}$, mentre quella estiva è di $144 \mathrm{~mm}$ (Bigi \& Rustici 1984). La presenza nell'area di corbezzolo, cisti ed erica arborea (Grifoni 2005) indica un influsso climatico mediterraneo.

Il substrato geologico dell'area è la Formazione di Londa (Oligocene), costituita da scisti siltosi e, subordinatamente, marne e arenarie fini quarzoso-feldspatiche e calcaree (Carta Geologica d'Italia 1967) ed ha originato suoli che, in seguito a rilievi pedologici di campagna effettuati secondo le indicazioni di Sanesi (1977), risultano da acidi a sub-acidi e privi di calcare.

I rilievi sono stati effettuati in 6 aree sperimentali permanenti di $400 \mathrm{~m}^{2}$, raggruppabili per composizione e struttura, in tre tipologie differenti: la prima, rappresentata in due particelle, è costituita da vecchi lembi di castagneto da frutto di scarsa fertilità nei quali la rovere, con età di 45 e di 41 anni, è stata inserita negli spazi creati dalla mortalità del castagno (cancro), ed ha via, via ridotto la presenza di quest'ultimo ad un piano dominato di polloni (tesi A); la seconda, costituita da tre particelle, si è formata inizialmente con l'inserimento della rovere in terreni liberi da vegetazione forestale, nei quali, a seguito delle utilizzazioni a ceduo e di un diradamento eseguito negli anni '70, si è formato un popolamento bi-stratificato di rovere, con un piano dominato e un piano dominante di 41 anni (tesi B); la terza, costituita da una particella con rovere di 45 anni di età inserita in un castagneto ceduo di scarsa fertilità, che con il tempo è divenuto piano dominato nei confronti della quercia (tesi C). Per ogni soprassuolo (Tab. 1) sono stati rilevati i caratteri stazionali (quota, esposizione, pendenza), l'età (nel 1996), il numero di piante di rovere per ettaro e l'altezza dominante (anno 1996). La valutazione qualitativa dei fusti è stata effettuata nell'anno 2000, applicando il metodo di stima della qualità delle piante in piedi messo a punto da Del Fave- ro et al. (1996). I parametri analizzati per la valutazione del fusto sono: altezza d'inserzione della chioma; assialità; numero di rami vivi, rami morti e rami epicormici; eccentricità; presenza di fibratura deviata; presenza di danni. Dai dati rilevati è stato poi ricavato un indice di qualità dei fusti (Del Favero et al. 1996). Fra il 1996 e il 1997, la proprietà ha effettuato interventi di diradamento con asportazione del piano dominato nelle particelle della tesi B; nell'anno 2000 , sulle piante di rovere rimaste è stato rilevato il numero di rami epicormici emessi dal tronco e definita la loro incidenza sull'indice di qualità del fusto. L'analisi statistica è stata effettuata con la procedura GLM (SAS 1996) considerando una ANOVA monofattoriale con il fattore "tesi" o il fattore "diradamento" per il confronto all'interno della tesi B.

\section{Risultati e discussione}

\section{Confronto fra tre tipologie di soprassuolo}

Le piante delle tre tesi presentano altezze di inserzione chioma, misurate con il "qualitometro" (Del Favero et al. 1996), analoghe e statisticamente non differenti. I valori rilevati, prossimi agli 8 metri di fusto (Tab. 2), sono, per la giovane età delle piante, di particolare interesse ed indicano che le condizioni pregresse del soprassuolo nelle 6 particelle hanno favorito l'autopotatura, determinando una discreta porzione di fusto potenzialmente utilizzabile. Il valore più basso di assialità del fusto $(5.63 \mathrm{~m})$ si registra nella tesi $\mathrm{A}$, risultato statisticamente differente rispetto a quelli riscontrati nelle altre due, che non risultano significativamente differenti fra loro: la B con $7.57 \mathrm{~m}$ e la C, la migliore, con $8.20 \mathrm{~m}$ (Tab. 2). Questo risultato può essere dovuto alle differenti condizioni di sviluppo in cui si sono trovate le piante nelle diverse tesi: nella $\mathrm{A}$, le roveri, inserite in posizione dominata rispetto alle piante di castagno, perlopiù da frutto, si 
Tab. 2 - Caratteristiche e indice di qualità dei fusti nelle tre tesi. $\left(^{*}\right)$ d.s.r. $=$ deviazione standard residua; $(a, b$, $c)=$ medie con lettere differenti sono statisticamente differenti $(\mathrm{p}<0.05)$.

\begin{tabular}{lcccc}
\hline \multicolumn{1}{c}{ Parametro } & tesi A & tesi B & tesi C & d.s.r. ${ }^{*}$ \\
\hline Altezza inserzione chioma (m) & 7.54 & 7.85 & 7.95 & 1.06 \\
Assialità fusto (m) & $5.63 \mathrm{a}$ & $7.57 \mathrm{~b}$ & $8.20 \mathrm{~b}$ & 1.61 \\
Rami morti fusto (n / fusto) & $1.63 \mathrm{a}$ & $2.82 \mathrm{~b}$ & $2.60 \mathrm{ab}$ & 1.95 \\
Rami vivi fusto (n / fusto) & $2.08 \mathrm{~b}$ & $1.15 \mathrm{a}$ & $2.80 \mathrm{~b}$ & 1.69 \\
Rami epicormici fusto (n / fusto) & 0.44 & 0 & 0.4 & 1.00 \\
Eccentricità fusto (D max / d min) & 1.07 & 1.08 & 1.08 & 0.04 \\
Fibratura deviata fusto (\%) & 0 & 0 & 0 & - \\
Danni al fusto (\%) & 12.50 & 2.70 & 0 & - \\
Indice qualità fusto (punti) & $15.66 \mathrm{a}$ & $17.08 \mathrm{~b}$ & $19.05 \mathrm{c}$ & 3.60 \\
\hline
\end{tabular}

sono sviluppate in maniera meno rettilinea, probabilmente perché impossibilitate ad allungarsi liberamente verso la luce; nella B le roveri, impiantate in terreni liberi da vegetazione forestale, hanno beneficiato fin dall'inizio anche della possibilità di farsi concorrenza senza impedimenti soprastanti e, infatti, presentano una maggior porzione di fusto diritto; nella tesi $\mathrm{C}$ le piante di rovere, inserite in un castagneto ceduo, hanno probabilmente usufruito delle condizioni migliori di concorrenza, tanto da presentare fusti lineari fin oltre gli $8 \mathrm{~m}$. In effetti, il ceduo di castagno per la sua rapida crescita non può essere il popolamento accessorio adatto (De Mas 1992), ma in questo caso una scarsa fertilità e problemi fitopatologici lo hanno, forse, reso meno concorrenziale nei confronti della rovere.

I rami morti (Tab. 2) sono meno presenti nelle piante della tesi A, dove, con un valore di 1.63 per fusto, risultano incidere statisticamente di meno rispetto alla tesi B e C (2.82 e 2.60 per fusto). Per una lettura complessiva della ramosità, è opportuno considerare anche i dati relativi ai rami vivi, che sono meno numerosi nella tesi $\mathrm{B}$, con 1.15 per fusto, e significativamente differenti rispetto a quelli della tesi A (2.08) e della tesi $\mathrm{C}$, dove ogni fusto presenta il massimo numero di rami vivi, 2.80 (Tab. 2). Questi dati sono di più difficile interpretazione, per le scarse notizie sugli interventi applicati ai soprassuoli negli anni precedenti l'ultimo decennio di analisi: per la tesi A si può supporre l'influenza positiva delle piante di castagno nel favorire l'autopotatura; analogo fenomeno è ipotizzabile per la tesi $\mathrm{B}$, dove la densità dei soprassuoli puri di rovere ha favorito una presenza ridotta di rami vivi e una notevole autopotatura, testimoniata dal discreto numero di rami morti lungo il tronco. Nella tesi $\mathrm{C}$, i più alti valori di ramosità morta e viva sono probabilmente da ricollegare ai tagli di utilizzazione effettuati periodicamente a carico del ceduo di castagno, che hanno esposto repentinamente alla luce i fusti di rovere, facilitando prima un'incremento di ramosità e, successivamente, una maggiore autopotatura a mano a mano che i polloni di castagno, con la loro rapida crescita, ne riombreggiavano i tronchi. I rami epicormici sono praticamente assenti in tutte le tesi: 0.44 per fusto nella tesi A, nessuno nella B e 0.4 nella C (Tab. 2). Quando gli epicormici spuntano precocemente possono, analogamente ai rami, indurre la formazione di nodi. I dati rilevati fanno ipotizzare un buon ombreggiamento del fusto sia da parte del soprassuolo principale che del piano dominato, costituito nelle tesi A e C da polloni di castagno e nella tesi B da polloni di rovere, dimostrando che un piano dominato costituito da specie non sciafile può, magari per periodi limitati, accompagnare positivamente i fusti del soprassuolo principale.

L' eccentricità del fusto delle piante di rovere, ricavata dal rapporto fra diametro massimo e minimo a $1.30 \mathrm{~m}$, non risulta differente nelle tre tesi (Tab. 2), attestandosi fra 1.07 (tesi A) e 1.08 (tesi B e C), valori quindi non superiori a quell'1.1 oltre il quale i tronchi sono considerati difettati (Del Favero et al., 1996). L'eccentricità nei fusti compare in aree pendenti o in presenza di ostacoli che ne rendano difficoltoso un corretto sviluppo (Del Favero et al., 1996); tuttavia, le differenti condizioni di pendenza delle particelle analizzate, spesso superiori ai $15^{\circ}$ (Tab. 1), non sembrerebbero aver influenzato questo importante parametro qualitativo.

Analizzando la direzione delle screpolature della 
Tab. 3 - Effetto del diradamento e dell'asportazione del piano dominato sull'emissione di rami epicormici e sulla variazione dell'indice di qualità dei fusti nella tesi B. $\left({ }^{*}\right)$ d.s.r. $=$ deviazione standard residua; $(a, b, c)=$ medie con lettere differenti sono statisticamente differenti $(p<0.05)$.

\begin{tabular}{ccccccc}
\hline \multirow{2}{*}{$\begin{array}{c}\text { Piante } \\
\text { Particella }\end{array}$} & $\begin{array}{c}\text { Eliminazione } \\
\text { asportate } \\
\text { piano }\end{array}$ & \multicolumn{2}{c}{$\begin{array}{c}\text { Rami epicormici } \\
\text { (n/fusto) }\end{array}$} & \multicolumn{2}{c}{ Indice di qualità } \\
\cline { 4 - 7 } dominato & Ante intervento & Post intervento & Ante intervento Post intervento \\
\hline 3 & 51 & SI & 0 & 18.38 a & 17.29 & 14.33 \\
4 & 47 & SI & 0 & $19.00 \mathrm{a}$ & 17.12 & 14.40 \\
5 & 43 & SI & 0 & $11.11 \mathrm{~b}$ & 16.89 & 14.71 \\
& & & & $10.75^{*}$ & $3.56^{*}$ & $3.63^{*}$ \\
\hline
\end{tabular}

corteccia, nelle tre tesi, non sono stati rilevati casi riconducibili al difetto della fibratura deviata (Tab. 2). Rare sono state le piante con danni rilevati nei fusti: nella tesi A il $12.5 \%$, tutti provocati da fulmini, il $2.7 \%$ nella tesi $\mathrm{B}$, procurati da interventi antropici, nessun danno nella tesi C. Questi due ultimi parametri non sono stati sottoposti ad elaborazione statisti$\mathrm{ca}$, trattandosi di frequenze sul totale delle piante osservate per tesi.

Per ogni tesi è stato calcolato, infine, l'indice medio di qualità dei fusti, per i quali, valutati come rovere da trancia, i campi di variazione di qualità individuati da Del Favero et al. (1996) sono: scadente, fino a 14 punti; buona, da 14 a 18 punti; ottima, da 19 a 24 punti. Seguendo la formula proposta (Del Favero et al. 1996) il risultato è stato di qualità buona nelle tesi A e $\mathrm{B}$, rispettivamente con 15.66 e 17.08 punti, e al limite inferiore della qualità ottima, con 19.05 punti, nella tesi C. I valori sono risultati, all'analisi statistica, significativamente diversi fra loro (Tab. 2).

\section{Effetto del diradamento e dell'asportazione del sottopiano sull'emissione dei rami epicormici}

Si è analizzato l'effetto della diversa entità del diradamento e dell'asportazione totale del piano dominato di rovere nelle tre particelle della tesi $\mathrm{B}$, i soprassuoli di rovere in purezza. Il diradamento ha asportato il $51 \%$, $47 \%$ e $43 \%$ delle piante, rispettivamente nelle particelle 3, 4 e 5. Prima del diradamento non erano presenti rami epicormici, mentre successivamente sono stati rilevati in media 18.38 rami di nuova emissione per ogni fusto nella particella più intensamente diradata, 19.00 nella particella a diradamento intermedio e 11.11 in quella meno diradata, dato quest'ultimo statisticamente differente rispetto agli altri due (Tab. 3). L'indice qualitativo medio di ciascuna particella, in seguito all'intervento, è quindi peggiorato, passando da 17.29 a 14.33 nella particella 3, da 17.12 a 14.40 nella 4 e da 16.89 a 14.71 nella 5
(Tab. 3), testimoniando diradamenti poco prudenti e l'infelice scelta di eliminare contemporaneamente il piano dominato di accompagnamento, in contrasto con le indicazioni suggerite da alcuni autori europei (Sevrin 1997, Hubert \& Courraud 1998).

In conclusione, dai dati raccolti emerge nei popolamenti esaminati che l'inserimento artificiale della rovere ha contribuito a formare soprassuoli diversi. Nei lembi di castagneto da frutto, oggi la quercia è divenuta dominante, ma con caratteri del fusto non ottimali, specialmente per l'assialità, probabilmente perché non sono state eliminate al momento giusto le piante di castagno soprastanti. I soprassuoli puri di rovere coetanei impiantati in terreni liberi da vegetazione forestale hanno fornito fusti di buona qualità, presumibilmente per la libera e reciproca competizione instauratasi. I fusti migliori di rovere sono stati individuati nel castagneto ceduo, sicuramente anche per l'azione educativa svolta dai polloni di castagno, meno competitivi del solito per la scarsa fertilità stazionale e per i problemi fitosanitari. Diradamenti di intensità compresa fra il $50 \%$ e il $43 \%$ e la contemporanea eliminazione del piano dominato, effettuati nelle particelle di rovere pura, hanno invece determinato un'emissione di rami epicormici che ha peggiorato l'indice di qualità dei fusti. I risultati confermano, dunque, che per produrre tronchi di rovere di qualità occorre applicare una selvicoltura puntuale e ben mirata.

\section{Ringraziamenti}

Si ringrazia il prof. Oreste Franci, della Facoltà di Agraria di Firenze, per la collaborazione fornita nell'elaborazione dei dati.

\section{Bibliografia}

Bigi L, Rustici L (1984). Regime idrico dei suoli e tipi climatici in Toscana. Regione Toscana, Firenze, pp. 129.

Carta Geologica d'Italia (1967). Foglio 114 (Arezzo), II edi- 
zione, scala 1:100.000. Servizio Geologico d'Italia.

De Mas G (1992). Primo contributo allo studio di tecniche per la coltura e la diffusione della rovere (Quercus petraea (Matt) Liebl) nei Colli Euganei (Padova). Vol. VI, 1984-91, pubblicazione n. 6, pp. 123-137.

Del Favero R, Abramo E, Zanella A (1996). La stima della qualità negli alberi in piedi. L'Italia Forestale e Montana, (6) 367-387.

Grifoni F (2005). Analisi dell'influenza della giacitura sullo sviluppo di soprassuoli artificiali di rovere (Quercus petraea (Matt) Liebl). Atti del IV Congresso Nazionale della Società Italiana di Selvicoltura ed Ecologia Forestale, S.I.S.E.F. Atti 4: 215-219.
Hofmann A, Goretti D, Merendi GA, Tabacchi G, Vignoli M, Bernetti G (1998). Boschi e macchie di Toscana 1. L'inventario forestale. Edizioni Regione Toscana, Firenze, pp. 219.

Hubert M, Courraud R (1998). Élagage et taille de formation des arbres forestiers. Edition Institut pour le Développement Forestier, Paris, pp. 303.

Sanesi G (1977). Guida alla descrizione del suolo. C.N.R., Firenze, pp. 157.

SAS (1996). SAS/STAT Software, release 6.12. SAS Institute Inc., Cary, NC, USA.

Sevrin E (1997). Les chênes sessile et pédonculé. Institut pour le Développement Forestier, Paris, pp. 96. 